\title{
Lol non tkt on ta pas oublié
}

Rapports à la norme et valeurs de la «faute » dans l'écriture SMS (projet et corpus Sud4science). Réflexions sociolinguistiques

Lol, non tkt on ta pas oublié. Sociolinguistic Considerations on SMS Writing Forms, in Relation to the French Standard and Spelling or Grammar Mistakes

\section{Claudine Moïse}

\section{(2) OpenEdition}

\section{Journals}

Édition électronique

URL : http://journals.openedition.org/pratiques/2721

DOI : $10.4000 /$ pratiques. 2721

ISSN : 2425-2042

Éditeur

Centre de recherche sur les médiations (CREM)

Référence électronique

Claudine Moïse, «Lol non tkt on ta pas oublié », Pratiques [En ligne], 167-168 | 2015, mis en ligne le 01 avril 2016, consulté le 19 avril 2019. URL : http://journals.openedition.org/pratiques/2721 ; DOI : $10.4000 /$ pratiques. 2721

Ce document a été généré automatiquement le 19 avril 2019.

(c) Tous droits réservés 


\title{
Lol non tkt on ta pas oublié
}

\author{
Rapports à la norme et valeurs de la « faute » dans l'écriture SMS (projet \\ et corpus Sud4science). Réflexions sociolinguistiques \\ Lol, non tkt on ta pas oublié. Sociolinguistic Considerations on SMS Writing \\ Forms, in Relation to the French Standard and Spelling or Grammar Mistakes
}

\section{Claudine Moïse}

1 Le lien avec la norme est au centre des perspectives sociolinguistiques, si l'on considère que la sociolinguistique rend compte des processus sociaux et donc des rapports de pouvoir et d'inégalité à travers les pratiques langagières (Fairclough, 1992, 2003; Bloemmaert, 2005 ; Heller, 2002). Ce rapport à la norme définit, en conséquence, les relations entre les langues, les enjeux diglossiques, l'insécurité linguistique, les discours idéologiques et donc épilinguistiques sur les langues. D'un point de vue des locuteurs et de ce que l'on pourrait appeler une sociolinguistique populaire ou une folk linguistic (Paveau, 2010), les discours épilinguistiques et axiologiques sur ce qui est du «bien et beau parler » ou du «mal parler », qui disent combien «le français se perd », « on parle de plus en plus mal », " les élèves font de plus en plus de fautes » font partie de la doxa sur les pratiques langagières (pour une synthèse, voir Moïse, 2009). Ils rendent compte et reproduisent des idéologies homogénéisantes sur la langue et la norme (Klinkenberg, 2001) dans le cadre d'un État-nation (Hobsbawm, 1990), expriment des peurs du changement, des moments de crises sociales ou servent la distinction sociale dans des rapports d'inclusion et d'exclusion (Barth, 1969). D'une manière comme une autre, ils traversent le temps et les modes de pensée avec passion (Paveau \& Rosier, 2008).

Si la réflexion sur la norme a ponctué la plupart de mes travaux en sociolinguistique interactionnelle, que ce soit autour de la description des parlers minoritaires en Ontario français au Canada, à travers les inscriptions urbaines, ou par l'usage transgressif des actes de langage dans la violence verbale, il me semblait que l'usage quotidien des SMS ${ }^{1}$, parce qu'il relève d'une pratique désormais répandue, utile voire ludique, pouvait peutêtre échapper aux carcans normatifs pour entrer dans une certaine modernité langagière qui dirait la diversité des usages, la liberté d'écrire et l'imaginaire scriptural. Or, les 
discours sur une orthographe, qui se verrait toujours plus détériorée par la pratique de l'écriture $\mathrm{SMS}^{2}$, et à contrario mon propre étonnement face à la diversité des pratiques d'écriture SMS (Panckhurst, 2012) ${ }^{3}$ m'ont amenée à me pencher sur la représentation de la norme à travers les textos ${ }^{4}$. Ainsi, à partir des réponses au questionnaire du projet Sud4Science et des commentaires métalinguistiques exprimés dans les textos, j'ai essayé de voir si les locuteurs reproduisent un discours normatif dominant ou si l'écriture SMS permet de développer un discours plus distancié et renouvelé par rapport à la norme.

\section{Un projet et les données ${ }^{5}$}

Dans le cadre du programme sud4science Languedoc-Roussillon. Mutation des pratiques scripturales en communication électronique médiée (en ligne : http://www.sud4science.org), coordonné par R. Panckhurst, lui-même s'insérant dans un vaste projet international intitulé sms4science (en ligne: http://www.sms4science.org; Fairon, Klein \& Paumier, 2006), nous ${ }^{6}$ avons constitué du 15 septembre au 15 décembre 2011, après suppression des doublons et des messages publicitaires, un corpus de 88522 SMS authentiques en langue française ${ }^{7}$. Sur 509 participants inscrits au départ, 424 ont livré des messages. L'objectif du projet, financé par la Maison des sciences de l'homme de Montpellier dans un premier temps et par la Délégation générale à la langue française et aux langues de France (DGLFLF) ensuite, a été, après anonymisation, transcodage et annotation partielle, d'effectuer des recherches pluridisciplinaires.

La collecte des SMS s'est faite par téléphone. Les participants qui voulaient « léguer leurs SMS à la science» devaient aller sur le site du projet et remplir une fiche de consentement ${ }^{8}$. La plupart des participants ont répondu au questionnaire proposé et très peu n'ont pas donné leur consentement (11 participants, qui ont donc été supprimés de la base définitive). Le questionnaire visait à la fois le type de téléphone utilisé, le choix des langues mais aussi les usages et les représentations liés aux SMS (opinions sur les SMS et avenir des SMS). Le corpus a été entièrement anonymisé à partir du logiciel Seek\&Hide - développé par deux étudiants en informatique (Accorsi et al., 2012 ; Patel et al., 2013) qui s'appuie sur des méthodes de traitement automatique du langage naturel (TALN). Le but du logiciel est de faciliter l'expertise et de traiter une quantité importante de données.

Un exemple d'anonymisation de prénoms complétée figure dans le tableau ci-dessous (cité in: Panckhurst et al., 2013) :

Tableau 1. Du SMS « brut » au SMS anonymisé (les chiffres renvoient au nombre de caractères du prénom dans le SMS brut)

Coco est pas la! Éva non plus ! Tanpis! Lol J’irai aux journée du patrimoine ! Éva m’a dit que tu venais cette semaine peut etre! Bisous!!

$<$ PRE_4 $>$ est pas la ! <PRE_3> non plus ! Tanpis! Lol J'irai aux journée du patrimoine ! < PRE_3> m'a dit que tu venais cette semaine peut etre ! Bisous!!

Une fois l'anonymisation terminée, les SMS sont prêts à être transcodés en français "standardisé » afin de permettre d'éventuels traitements ultérieurs en linguistiqueinformatique (incluant des analyseurs syntaxiques). 
$<\mathrm{PRE}_{-} 4>$ est pas là ! <PRE_3> non plus! Tant pis! Lol. J'irai aux journée s du patrimoine! $<\mathrm{PRE}_{-} 3>$ m'a dit que tu venais cette semaine peut-être ! Bisous ! !

4 Enfin, une phase d'annotation optionnelle prévoit l'utilisation de huit étiquettes: ABSence, BINettes, DIVers, GRAmmaire, LANgage, MODification, ORThographe, TYPographie. Quatre de ces étiquettes sont utilisées dans le tableau 3 ci-dessous (un double étiquetage peut être employé en cas d'ambigüité) :

Tableau 3. SMS annoté (étiquettes indiquées en gras)

<PRE_4> <ABS_n'> est pas <TYP_>là! <PRE_3> non plus! <MOD_t_TYP_space>Tant pis! $<$ MOD_laughing out loud $>$ Lol $<$ TYP_.>. J'irai aux <GRA_MOD>journées du patrimoine! $<\mathrm{PRE}_{-} 3>$ m'a dit que tu venais cette semaine $<$ TYP__-^>peut-être ! Bisous! !

5 Mon analyse s'appuie donc ici à la fois sur les réponses au questionnaire et sur quelques exemples de SMS du corpus 88milSMS.

\section{La norme linguistique et l'idéologie de la nation ${ }^{9}$}

Dans une perspective sociolinguistique, critique et interactionnelle, comme je l'ai mentionné, l'analyse des rapports de pouvoir et donc des rapports à la norme sont au centre de ma réflexion. La construction idéologique de la norme repose sur le contrôle social et l'ordre public. La langue française est au fondement de la République (article 1. de la Constitution française, La France est une République indivisible, laïque, démocratique et sociale; et article 2. La langue de la République est le français) et a constitué historiquement un corps social (Balibar, 1985). À travers les fondements idéologiques d'une nation unifiée et unificatrice (universalisme, raison, unité), la langue française peut alors jouer du contrôle social. Elle est donc, dans ses formes et sa gestion, de l'ordre du politique, qui se légitime par elle, comme elle est légitimée par lui ; elle devient symbole du pouvoir, inscrite dans la constitution et partagée par la nation (Eloy, 2000 : 107). La Révolution française et la Troisième République ont continué d'user de la langue française pour construire le citoyen et définir un espace public égalitaire, unificateur et homogène ; dans cette optique la langue, à travers l'école reproductrice des valeurs républicaines, a épousé les vertus laïques (Dubet, 1997). La laïcité, à l'image de toute religion, se voit relayée par le livre sacré qu'est le Dictionnaire (Cerquiglini, 2003) et ses vertus sacralisées s'actualisent dans les valeurs morales et civiques attribuées à la langue française (Gadet 2003): l'espace public se construit en français, dans l'apprentissage de la difficulté linguistique où «ne pas faire de fautes » devient signe distinctif et force émancipatrice. L'accès alors au "bon français ", à travers l'idée de démocratie, via l'égalité et la méritocratie, sert par là même la reproduction de l'élite, sous critère de distinction. La norme, bien commun national, se fait marque de reconnaissance.

Même si la réalité des langues est dans le multiple, la construction centralisatrice de l'ordre national réaffirme sans cesse l'unité républicaine en langue française, contre les 
réalités hétérogènes. Face à une telle construction idéologique et linguistique, toute menace ressentie à l'égard de la langue française participe d'une déstabilisation du corps social et politique, et inversement. Il n'est pas étonnant alors que, dans une telle optique, les métaphores de la menace se perpétuent de siècle en siècle, depuis les récriminations contre les emprunts à l'italien au XVI ${ }^{e}$ siècle, jusqu'aujourd'hui dans les livres de bon usage, dans la presse ou dans les chroniques des lecteurs. L'évocation de «la crise du français » traverse le XIX ${ }^{e}$ siècle et joue des mêmes lamentos ${ }^{10}$. Même s'il est difficile voire impossible de répondre de façon objective en diachronie au sujet d'une perte de la qualité en langue (qu'est-ce que la «qualité »?, Eloy 1995) face à l'évolution linguistique (quels seraient les critères objectifs d'analyse pour une perte de qualité ?), il est certain que l'image de la pureté d'une langue homogène qui se perdrait est une peur ancienne, éminemment subjective. Cette crainte porte à la fois sur le maintien d'une unicité de la langue française contre toute pluralité et sur son intégrité, essentiellement à travers la part la plus flexible du système, le lexique ${ }^{11}$. Au thème de la pureté vient donc se greffer le discours sur le manque de qualité du français et sur son appauvrissement, marqué particulièrement, d'hier à aujourd'hui, par l'insuffisance du vocabulaire des jeunes et par la méconnaissance de l'orthographe. Déjà C. Bally en 1930, face aux imprécations contre la langue, de sa corruption à sa perte de valeurs, critiquait le mécanisme de «crise de la langue ». Si les changements en langue, morphologie et syntaxe, se font très lentement, C. Bally $(2004,: 18)$ montre combien la nouveauté lexicale affecte les émotions et « voilà ce qui alarme et indigne et quand la tempête fait déborder le verre d'eau, on parle de crise du français». Depuis, dans sa trace, maints linguistes (Meschonnic, 1997; Klinkenberg, 1993) ont montré combien, des figures de l'élite à l'opinion publique et à la presse, on n'en finissait pas d'accuser les pédagogies laxistes, les programmes allégés ou une baisse du niveau scolaire.

Les discours sur les SMS font ainsi écho à d'autres discours qui ont traversé l'histoire. « La dénonciation des "fautes" est le propre du passé comme du présent. Sous la restauration comme aujourd'hui, les bourgeois assurés dans leur français écrit ne manquent pas de rire des gens du peuple, puisque, maintenant, ils savent écrire. Et ils parlent mal puisqu'ils parlent un peu autrement : on les renvoie à la grammaire et au dictionnaire » (Rey, $2007: 275-277$ ). Ainsi, M. Alhoy décrit dans ses textes ${ }^{12}$ l'orthographe des femmes du peuple: «Il y a des Lorettes qui poussent jusqu'à l'avarice la plus sordide, l'économie qu'elles font des lettres de l'alphabet. Elles écrivent $J$ rai vous voir. Et si vous leur demandez pourquoi elles suppriment dans “j'irai” l'apostrophe et l'i elles disent que c'est de trop que ça ne change rien au mot. Elles écrivent aussi $G$ dîné. Mon chair, je croyai que 7 es pour de main ${ }^{13} »$.

\section{Analyses des jugements sur l'écriture SMS}

7 Les analyses portent sur les déclarations recueillies dans le questionnaire, à partir de la question « D'après vous quels sont les avantages / inconvénients de l'écriture SMS ? » ${ }^{14}$. Je n'ai tenu compte, dans les résultats présentés ici, que des participants qui avaient répondu exclusivement soit sur des avantages, soit sur des inconvénients. 


\subsection{Les avantages des SMS}

8 Les profils scolaires des participants qui expriment les avantages (au total, 71) offerts par l'écriture SMS sont très variés (31 études supérieures, 7 niveau CAP, 6 adultes bac, 13 lycée, 13 collège) et traversent donc les générations et les niveaux d'étude.

Les avantages mentionnés portent non pas sur la langue, mais essentiellement sur les pratiques conversationnelles. Elles se caractérisent ainsi par leur rapidité (39 occurrences), mais aussi par leur discrétion (8 occurrences) [ben on peut dire mor de rire $m d r$ ou lol $e$ on entan pa votre conversation -12 ans, M, collège], l'asynchronie (6 occurrences) [Ils sont très pratiques car ils me permettent de faire passer un message sans déranger la personne. On doit répondre tout de suite (plus ou moins) à un appel alors que nous pouvons répondre aux sms quand nous voulons -20 ans, F, bac +3 ], leur aspect ludique (3 occurrences) [Le sms est aussi pratique quand on n'a pas forcément quelque chose à dire, que l'on veut juste s'occuper ou s'amuser un peu. Au téléphone, appeler pour ne rien dire, c'est un peu risqué -22 ans, $\mathrm{F}$, bac +4$]$. L'usage des SMS permet aussi de laisser une trace (3 occurrences) [Les avantages des sms sont que nous pouvons joindre une personne sans forcément la déranger car elle peut lire le sms quand elle le désire \& que le message "reste » ce qui est pratique pour communiquer une adresse par exemple - 22 ans, F, bac + 3] ; les SMS sont un élément de désinhibition (3 occurrences) [cé plu facile pour communiquer surtout pour les timide -19 ans, F, bac +1 ], ils créent le lien (3 occurrences) [Ils permettent de rester en contact - 15 ans, M, lycée].

Ces maintiens du lien qui tissent la relation ${ }^{15}$ sont largement exprimés à travers les SMS eux-mêmes, qui marquent bien souvent «la salutation, le souhait, le témoignage d'affection, le remerciement, le compliment ou la prise de congé » (Vérine, 2013) : [Kikou ma petite $\left\langle P R E_{-} 6>\right.$ ça va? Juste un petit SMS pour souhaiter a ta Maman un joyeux anniversaire!!! ;) bonne journée!] [oublie surtout pas mes petits sms de la journée:\$ <3 je t’aime chaton], [Je t'en prie ;) ma journée est déjà ensoleillé avec ton SMS :) merci! Bonne journée a toi aussi. Gros gros bisous a tous les deux!!!! ${ }^{16}$.

Au-delà des pratiques conversationnelles, les SMS jouent sur les codes et rituels conversationnels en les simplifiant [L'envoi de sms permet de communiquer quasi instantanément en allant directement au but, contrairement à la conversation téléphonique qui induit un certain nombre de passages obligés avant d'entamer le sujet réel de conversation. - 22 ans, F, bac + 4], en maniant expressivité [c'est rapide mais avec les SMS c'est de donner un ton par exemple si c 'est rigolo ou ironique. -28 ans, F, bac +2 ], jeux avec les mots [c'est un jeu avec les mots et la phonétique. -65 ans, $\mathrm{M}, \mathrm{bac}+4$ ], style [lécriture sms nous permet de montrer notre style que nous aimons -28 ans, M, bac +4 ], [Quand on reçoit un SMS un n'attend pas forcément que l'écriture soit correcte - 18 ans, F, bac + 1].

\subsection{Les inconvénients des SMS}

9 Le nombre total de mots pour les avantages cités était de 852 mots pour 70 personnes (soit une moyenne de 12,1 mots). Pour les inconvénients, on voit le nombre de mots croitre de façon significative et passer à 1324 pour 82 personnes (soit une moyenne de 16,1 mots). Comme on le voit souvent, émettre des critiques est plus facile que lister des avantages, sans doute parce que les émotions négatives marquent davantage que celles positives et que le besoin d'exprimer ses frustrations et ses manques est plus fort que la nécessité de dire ses contentements (Luminet, 2002). 
Pour les profils des participants, on trouve davantage de classes supérieures (44 études supérieures, 10 adultes bac, 2 niveau CAP, 1 adulte niveau primaire, 15 lycée, 9 collège) qui critiquent l'usage de l'écriture SMS, ce qui peut s'expliquer peut-être par une plus forte conscience des pratiques normatives et un besoin de distinction.

Cette fois-ci les inconvénients ne portent pas vraiment sur les pratiques conversationnelles. Le sens d'« écriture SMS », qui avait été compris comme «échanges par SMS", laisse la place ici à celui de "graphie SMS». Parmi les pratiques conversationnelles qui sont critiquées, on peut mentionner, l'écran tactile qui entrave l'écriture [avec l'ecran tactile faut regarder ce que l'on tape - 27 ans, M, bac], l'échange in absentia avec l'allocutaire [On ne sait jamais ce que pense réellement le destinataire... Les gens ne voient pas la tête que l'on fait ou ne peuvent entendre l'intonation ... - 19 ans, F, bac + 1] qui entraine parfois des malentendus conversationnels, souvent mentionnés (8 occurrences), [Parfois on a du mal à comprendre ce que la personne veut nous dire qd c'est de l'humour par exemple - 33 ans, F, niveau supérieur], [l'écrit peut être mal perçu : un simple "ok" peut vouloir à la fois dire "c'est bon tu me gonfles " ou "j'ai bien compris" - 41 ans, F, bac + 4], [le fait de ne pas pouvoir mettre de ton dans les phrases. Suivant la personne ça peut être mal interprété -19 ans, $\mathrm{F}, \mathrm{bac}+1]$.

L'écriture elle-même peut entraver la compréhension des messages, comme il est souvent relevé (14 occurrences) [il arrive qu'ont comprenne pas tout celon l age de la personne et la rapidité dont c 'est ecris - 41 ans, F, CAP].

Mais les occurrences les plus nombreuses concernent le jugement porté à la langue. On trouve 11 occurrences du mot «faute " [trop de fautes - 19 ans, F, bac + 2], 21 occurrences du mot " orthographe », 13 de " perte » [L'inconvénient de l'écriture Sms est sans conteste la perte du niveau d'orthographe - 20 ans, F, bac + 2] ( " perte du niveau d'orthographe », «la perte de l'écriture française ", "perte de l'orthographe ", "une perte de la langue française », "perte écriture française ", " on perd les notions d'orthographe et de conjugaison ", «la langue française se perd ", " aucun à part de perdre toute notion du français »).

De façon plus générale, on relève 24 occurrences qui relatent la «détérioration de la langue » (je souligne) [On ne sait plus écrire comme il se doit - 20 ans, F, bac + 2], [de mauvais rêflexes qui se répercuteront dans la pauvreté du langage oral et/ou rédactionnel - 21 ans, $\mathrm{M}$, bac +5], [On perd l'habitude d'écrire réellement et correctement notre langue maternelle - 18 ans, F, $\mathrm{bac}+2]$, [Elle détruit progressivement l'orthographe et sa richesse -23 ans, F, bac +4$]$, [le principal inconvénient de l'écriture sms est que les jeunes ne savent plus écrire correctement - 34 ans, F, bac + 4], [L'orthographe et la grammaire française disparaissent malheureusement... - 19 ans, F, bac], [ça gache la langue française, - 17 ans, F, lycée], [L'écriture sms $n$ 'a aucun avantage et peut même être considéré comme un fléau. Cela appauvri notre langue - 21 ans, $\mathrm{M}$, bac + 2], [C'est moins agréable à lire qu'un SMS bien rédigé - 16 ans, F, bac], [Peut entraîner de fortes confusions orthographiques pour l'écriture "normale" de mails, de courriers, etc.- 30 ans F bac + 5], [ $L$ 'écriture SMS est de plus en plus moquée. Elle est parfois ilisible et $n$ 'apporte rien - 17 ans, $\mathrm{F}$, lycée].

Cette détérioration de la langue touche aussi par ricochet social son utilisateur [Ce n'est pas beau et donne un style "illettré" - 16 ans, $\mathrm{M}$, lycée], [Aujourd'hui avec un accès facile je trouve que sa fait débile - 15 ans, $\mathrm{M}$, lycée], [Moins esthétique que la "vraie langue [...] c'est une régression culturelle... -52 ans, $\mathrm{M}, \mathrm{bac}+8$ ], [petit avantage: c'est plus rapide TRES GROS INCONVENIENT : le Monde ne saura plus écrire le Français - 51 ans, F, CAP] 


\subsection{Un discours circulant}

11 Ainsi, les principales critiques, et même des plus jeunes, portent sur la perte qui s'exprime en termes de manque : manque de rigueur (« comme il se doit», «le Monde ne saura plus écrire le Français $[s i c] »)$, manque de justesse («écrire correctement»), manque de richesse (" pauvreté du langage »), manque de beauté (" ça gâche », « moins esthétique »), manque de culture (« donne un style « illettré », « fait débile », « régression culturelle »). L'écriture SMS, en ce sens, serait un «fléau ». Ces sentiments de pureté et de distinction sociale que nous avons évoqués s'appuient sur une forte impression de menace qui dit avant tout "la puissance de résistance aux changements" (Chiss \& Puech, 2004). La "crise de la langue » ne s'appuie pas sur un dysfonctionnement de l'usage de la langue, c'est-à-dire de sa faculté instrumentale de communication, mais sur sa portée symbolique. Les évolutions phonologiques, morphologiques ou sémantiques sont minimes au regard d'une génération (Klinkenberg, 1993: 178-179), mais l'impression de dégradation est à mettre en lien avec des crises sociales et des formes d'insécurisation. Parce que la langue renverrait à une identification de soi, toute période de l'histoire faite de mutations s'accompagnerait d'un certain désarroi et de fragilités projetés en langue (Klinkenberg, 1993). Les différences en langue, le français qui se démocratise et «s'encanaille " (Bally, $2004: 24)$, ont pour effet de provoquer des craintes de fragilisation de la langue de référence, celle représentée par une certaine norme idéalisée, portée par des élites à la légitimité menacée, dans un effet de remise en question de l'ordre social. «La crise du français est une crise de classe » (Meschonnic, 1997 : 286) et renvoie donc à des pertes de pouvoir ${ }^{17}$ (Vernet, 2010), de sécurité et de stabilité. En ce sens, le discours sur la langue et sur les menaces qu'elle encourt, discours ancestral, est très fortement porté par un bouleversement idéologique. La crise de la langue n'est "qu'un aspect particulier d'un phénomène plus vaste: celui d'un effondrement des valeurs ", pris dans une crise de "conscience» ou de "confiance» (Klinkenberg, 2001: 109-110) qui, du malaise culturel, politique et social résonne en langue comme si le sentiment de la crise $\mathrm{du}$ français était aussi le symptôme d'une crise nationale, identitaire et culturelle. Ce discours de la crise ou de la perte circule alors, sous forme de doxa, du milieu scolaire aux médias, repris jusque par les plus jeunes sous formes de collocations presque figées voire stéréotypées qui reproduisent et alimentent les représentations. Alors que l'on n'a jamais autant écrit, que l'on échange énormément par SMS et que les différentes façons d'écrire semblent acceptées dans les échanges ${ }^{18}$, le discours sur le manque paraissent s'inscrire dans des effets d'évidence non questionnés.

\subsection{Un discours pathémique}

La construction argumentative, dans le but non seulement de démontrer mais de convaincre, sur les inconvénients de l'écriture SMS est relativement pathémique et use de plusieurs procédés qui vont au-delà de simples faits informatifs. L'axiologie négative s'appuie sur l'évocation d'émotions, telles la compassion, la désolation ou la tristesse, belle façon de rallier ceux à qui l'on s'adresse et, par là, de reproduire cette idéologie de la perte par un effet de « contagion émotionnelle » (Rimé \& Moscovici, 2005).

Ainsi, on trouve le recours à des subjectivèmes négatifs adjectivaux (je souligne) [le français se perd certaines informations très importantes sont dites par écrit au lieu d'être annoncées oralement, c'est triste.... - 38 ans, F, bac + 3], [L'orthographe et la grammaire française 
disparaissent malheureusement... - 19 ans, $F$, bac], à un groupe porteur de la faute, sorte de bouc émissaire, contre lequel on peut faire alliance dans un effet de stigmatisation et d'exclusion bien connu, les «jeunes » en l'occurrence [le principal inconvénient de l'écriture sms est que les jeunes ne savent plus écrire correctement -34 ans, F, bac +4$]$, [Cela fait faire énormément de fautes d 'orthographe surtout pour les plus jeunes... - 28 ans, F, bac + 3], [C 'est illisible, ridicule, enlève toute crédibilité à l'interlocuteur et donne aux adultes une image pitoyable de l'adolescence - 18 ans, $\mathrm{M}$, lycée], à la l'adresse à l'auditoire [Je $m$ 'arrêterai là pour ne pas tomber dans la vulgarité. Sachez seulement que tous mes amis pensent comme moi, et que si je reçois un SMS écrit ainsi, je le supprime sans même le lire -18 ans, M, lycée], à l'exemplification par la narration [Au début, quand j'étais au collège j'écrivais en "langage " sms et j'ai eu 1 à une dictée Je ne sais pas si c'est vraiment ça qui m'a fait échouer mais j'ai quand même tout de suite arrêté Depuis je ne fais pratiquement plus aucune faute - 17 ans, F, bac].

Enfin, contrairement à l'expression des avantages où la source énonciative, n'est pas prise en charge par un «je ", mais soit par un «nous » englobant [l'écriture sms nous permet de montrer notre style que nous aimons - 28 ans, $\mathrm{M}, \mathrm{bac}+4$ ], soit par un « on » inclusif [Le sms est rapide. Il peut-être envoyé et reçu discrètement. On peut s'attendre à ce que la personne nous réponde à tout moment alors que l'appel sauf si on laisse un message (c'est plus long et contrariant d'écouter sa boite vocale. Je sais que moi je n 'aime pas) la personne ne peut réponde que s'il est disponible, s'il elle a le téléphone avec elle, si elle a entendu sonner - 23 ans, F, bac + 5] qui permettent à la fois généralisation et distanciation, l'expression des désavantages, et notamment ceux qui ont trait à la langue, se manifeste à travers la prise en charge par un « je » énonciatif qui affirme fortement une prise de position dans une mise en narration possible: [Je crains une dégradation de la langue et del'orthographe et estime que les jeunes et moins jeunes considèrent trop l'orthographe comme inutile. -53 ans, $\mathrm{M}, \mathrm{bac}+8$ ], [selon moi, l'écriture SMS apporte plus d'inconvénients que d'avantages. Déjà, les gens (surtout les jeunes) ne savent plus écrire. Il est très désagréable de lire des mails, des lettres (ou autres) remplis de fautes d'orthographe. La langue française est une magnifique langue, et je trouve cela dommage de la ternir par l'écriture SMS. Par ailleurs, cela va pénaliser beaucoup de monde, particulièrement les élèves. Un jour, par exemple, un professeur d'hitoire-géograhpie nous a rendu des copies. Il était horrifié, car sur l'une d 'entre elle était marqué D3 (pour détroit). 'élève ne l 'avait même pas fait exprès! C'est assez troublant... Ensuite, il me faut parfois déchiffrer les textos que l'on $m$ 'envoie en langage sms. ça ne me donne même pas envie de le lire, et encore moins d'y répondre! Je suis très attachée à ce que tout soit bien écrit (avec les accents, la ponctuation, etc.). Il faut dire que, depuis que j'ai mon portable, les textos sont un peu comme une boíte mail, pour moi. Ayant un forfait sms illimités, je peux me permettre d'envoyer des messages très longs, comme si c 'était de vrais mails. Pour moi, c'est une forme de respect d'écrire correctement à la personne à qui j'envoie un message. Ce que je constate, c'est que plus les jeunes ont un portable tôt, moins ils savent s'exprimer correctement à l 'écrit. Personnellement, je n'ai eu mon portable qu'après avoir passé mon Bac, et je me rends compte que c 'est une véritable chance :je n 'ai pas baigné, dès petite, dans ce milieu où la langue française est abîmée, alors qu'elle est si riche. Je désapprouve totalement le fait que mes parents écrivent eux-mêmes en langage sms. Cela n'incitera jamais mes petits frères à écrire normalement avec leur portable... -19 ans, $\mathrm{F}, \mathrm{bac}+2]$.

\section{En guise de conclusion}

Comme il a été montré ailleurs, l'écriture SMS est riche (Panckhurst, 2014) de ses néologismes, de ses mots d'adresses/mots doux ${ }^{19}$ (Détrie \& Vérine, 2012), de ses binettes 
pour dire les émotions ${ }^{20}$ et de ses créations graphiques ${ }^{21}$ (pour une typologie voir Panckhurst, 2009). Elle permet, au-delà des erreurs orthographiques qui peuvent être repérées et qui sont de tous les écrits, des cartes postales de la guerre de 14-18 aux notes de travail, de jouer sur les graphies et sur la langue. L'écriture SMS s'amuse des variations, des effets poétique ${ }^{22}$ ludique et apocoristique ${ }^{23}$; elle sert la connivence, elle entretient les liens intimes et joue un rôle informatif, rapide et direct. Malgré ce constat, on constate que les représentations sur le bien-écrire traversent encore les discours, même chez les plus jeunes, où s'exprime non pas un éloge de la variation stylistique par exemple, mais un idéal de la norme qui, face à une détérioration supposée de la langue, sert, encore une fois, une image de la pureté contre la perte de la qualité.

\section{BIBLIOGRAPHIE}

ACCORSI, P., PATEL, N., LOPEZ, C., PANCKHURST, R. \& ROCHE, M. (2012). « Seek\&Hide: Anonymising a French SMS corpus using natural language processing techniques ». Lingvistica Investigationes, special Issue 35, 2, p. 163-180

BALIBAR, R. 1985, L'Institution du français. Paris, Presses universitaires de France.

BALLY, C. (2004) [1930]. La Crise du français. Genève : Droz.

BARTH, F. (1969). « Introduction ». In: Barth, F. (ed.), Ethnic Groupes and Boundaries. The Social Organization of Culture Difference. Boston, Little, Brown and Co, p. 9-38

BERNicot, J., GOUMI, A., BERT-ERboul, A., \& VolCKAERT-LEgRier, O. (2014). « How do skilled and lessskilled spellers write text messages? A longitudinal study », Journal of Computer Assisted Learning Blommaert, J. (2005). Discourse. Cambridge, Cambridge University Press

CERQUiGLINI, B. (2003). « Le français, religion d'État ». Le Monde, 25 nov. 2003.

CHISS, J.-L. \& PUECH, C. (2004) [1930]. « De l'usage de la crise en matière linguistique ». In: Bally, C., La Crise du français. Genève : Droz, p. 94-114.

DÉTRIE, C. \& VERINE, B. (2012). «Quand l'insulte se fait mot doux : la violence verbale dans les SMS ». Colloquium Dimensions du dialogisme 3 : Du malentendu à la violence verbale. Helsinki, Finlande, 15-17 aout 2012.

DUBET, F. (1997). « La laïcité dans les mutations de l'école ». In: Wieviorka, M. (éd.), Une société fragmentée ? Le multiculturalisme en débat, Paris : La Découverte, p. 85-112

ELOY, J.-M. (éd.) (1995). La Qualité de la langue française ? Le cas du français. Paris : Champion. - (2000). « G, comme gérer la langue ». In: Cerquiglini, B., Corbeil, J.-C., Klinkenberg, J.-M. \& Peeters, B. (eds), « Tu parles! ?Le français dans tous ses états. Paris : Flammarion, p. 95-107.

GADET, F. (2003). La Variation sociale en français. Orphys, Paris.

FAIRCLOUgh, N. (1992). Discourse and Social Change. Cambridge, Polity Press.

- (2003). Analysing Discourse : Textual Analysis for Social Research. Londres, Routledge. 
FAIRON, C., KLEIN, J.-R. \& PAUMIER, S. (2006). SMS pour la science. Corpus de 30.000 SMS et logiciel de consultation. Manuel + cédérom. Louvain-la-Neuve : Presses universitaires de Louvain. En ligne : http://www.smspourlascience.be/ (consulté le 01/04/16).

HOBSBAWN, E. (1990). Nations and Nationalism since 1780: Programm, Myth, Reality. Cambridge : Cambridge University Press.

HELLER, M. (2002). Éléments d'une sociolinguistique critique, Paris, Didier.

KLINKENBERG, J.-M. (1993). « Le français : une langue en crise ? ». Études françaises 29-1, p. 172-190. - 2001, La Langue et le citoyen. Paris, Presses universitaires de France.

LUMINET, O. (2002). Psychologie des émotions. Confrontation et évitement. Bruxelles : De Boeck.

MESCHONNIC, H.,(1997). De la langue française. Paris : Hachette.

MOÏSE, C. (2009). « Le poids de la langue française, entre sentiment de menace et dynamiques langagières ». In: Gasquet-Cyrus, M. \& Petitjean, C. (éds), Le Poids des langues. Paris : L'Harmattan, p. 237-253.

PANCKHURST, R. (2009). « Short Message Service (SMS) : typologie et problématiques futures ». In: Arnavielle, T. (éd.), Polyphonies, pour Michelle Lanvin. Montpellier : Université Paul-Valéry Montpellier 3, p. 33-52. En ligne : http://hal.archives-ouvertes.fr/hal-00443014 (consulté le 01/04/16).

- (2012). « sud4science Languedoc-Roussillon : collecte et analyse de 90000 SMS authentiques ». Invited talk. Mons : La Fabrique, 27 avril. En ligne : http://www.aprem-exploration1.net/ archives-exploration1-le-labophone.html (consulté le 01/04/16).

- (2014). « 88milSMS : l'écrit quotidien dans les SMS ». Conférence. Université Paris-Sorbonne, mai 2014.

PANCKHURST, R., DÉTRIE, C., LOPEZ, C., MOÏSE, C., ROCHE \& M., VERINE, B. (2013). « Sud4science, de l'acquisition d'un grand corpus de SMS en français à l'analyse de l'écriture SMS ». Epistémé 9 , p. 107-138.

PATEL, N., ACCORSI, P., INKPEN, D., LOPEZ, C. \& ROCHE, M. (2013). « Approaches of anonymisation of an SMS corpus ». In: Proceedings of CICLING (Conference on Intelligent Text Processing and Computational Linguistics). Berlin: Springer : 77-88. En ligne : http://www.cicling.org/2013/ (consulté le 01/04/16).

PAVEAU, M.-A. (2010). « Do non-linguists practice linguistics? An anti-eliminative approach to folk theories ». AILA Review 24, p. 40-54.

PAVEAU, M.-A. \& Rosier, L. (2008). La Langue française. Passion et polémiques. Paris, Vuibert.

REY, A. (2007). L'Amour du français : contre les puristes et autres censeurs de la langue, Paris, Denoël.

RIMÉ, B. \& MoscovicI, S. (2005). Le Partage social des émotions. Paris : Presses universitaires de France.

VERINE, B. (2013). « Les verbes SMS, texto, texter et textoter dans le corpus sud4science ». En ligne : http://praxiling.hypotheses.org/348 (consulté le 01/04/16).

VERNET, S. (2010). Rapport à la norme : discours métalinguistique spontané sur des forums anglophones et francophones. Mémoire de master 2, Grenoble 3. En ligne : http://dumas.ccsd.cnrs.fr/ docs/00/70/48/79/PDF/VERNET_Samuel_M2R.pdf (consulté le 01/04/16). 


\section{NOTES}

1. SMS signifie « short message service » devenu « services de messages succincts » en français.

2. Malgré les études, telle celle réalisée par le Centre de recherche sur la cognition et l'apprentissage (Bernicot et al., 2014) qui montrent que l'écriture SMS n'a pas d'incidence sur l'écriture des adolescents, les discours qui vont à l'encontre de ce constat, notamment sur certains blogs et forums de discussion sur Internet, sont nombreux.

3. Des SMS comme (1) [wesh trkl tkt;) tu fou quoi ?], (2) [Oh ban nan!ce week end c'est bon! Faut que je fasse des courses d'habits], (3) [Ta u <PRE 5> o tel?], (4) [As tu u le tps de fer le virment $a<P R E \_3>$ ? ], (5) [ j'ai fait quelques recherches: ma table est probablement une table à téléphone ou une table à thé dans un style Louis XIII (ou XVIIe s.) revisité. Il faut savoir que le style Louis XIII était considéré bucolique au XIXe et au début du XXe s. Je suis assez catégorique : des éléments architecturaux XVIIe s. mais aux dimensions graciles très 1920. Par contre l'origine...] font état de cette diversité. Les messages (1) et (2) ont été envoyés par des élèves de collège âgés de 12 ans, les messages (3) et (4), peut-être contre toute attente par une femme à la retraite de 57 ans, et de 5 ans d'université et pour le message (5) un jeune homme de 24 ans avec 4 ans d'université.

4. Dans le corpus Sud4science, on trouve 503 occurrences de SMS pour 437 de la famille lexicale de texto (Vérine, 2013).

5. Pour une présentation détaillée des différentes étapes du projet, voir R. Panckhurst et al. (2013).

6. Ce projet est le résultat d'un travail d'équipe comprenant Rachel Panckhurst (responsable du projet, Tal), Catherine Détrie (analyse de discours), Cédric Lopez (informaticien), Claudine Moïse (sociolinguistique interactionnelle), Mathieu Roche (informaticien) et Bertrand Vérine (analyse de discours). Je tiens aussi à remercier les étudiants de M1 de Sciences du Langage à Montpellier 3 qui ont analysé en partie le questionnaire, et les stagiaires qui ont travaillé à l'anonymisation et au transcodage du corpus.

7. Le corpus final s'intitule 88milsMS

8. Cette étape a demandé un gros travail d'élaboration, notamment parce qu'il a fallu tenir compte des droits et des libertés, surtout pour les mineurs.

9. Je reprends ici les grandes idées de C. Moïse (2009).

10. J.-M. Klinkenberg (1993) s'amuse à répertorier les ouvrages qui, tout au long du XIX ${ }^{\mathrm{e}}$ siècle, ont ponctué «la crise du français» pour montrer combien ils jouent sur les mêmes récriminations, essentiellement portant sur la perte de qualité du français, sur sa mort prochaine, sur son mauvais enseignement.

11. La loi Toubon de 1994, sur «l'emploi de la langue française » dans les affiches publicitaires, les contrats de travail et les modes d'emploi notamment, s'inscrivait de fait contre les anglicismes, symboles d'impureté en langue.

12. Il s'agit de petits ouvrages, « les physiologies », qui visent une catégorie sociale parisienne et féminine dans une forme discriminante et sexiste, dite La Lorette. En ligne : http://gallica.bnf.fr/ ark:/12148/bpt6k1132844/f21.image (consulté le 01/40/16).

13. En ligne : http://gallica.bnf.fr/ark:/12148/bpt6k1132844/f62.image (consulté le 01/04/16).

14. 350 personnes ont répondu au questionnaire parmi les 423 participants au projet. Aucun questionnaire n'a été supprimé.

15. «On relève par ailleurs une dizaine de variations sur dire / discuter / parler par SMS ou par texto, et de nombreux messages programmant ou commentant des stratégies de séduction amoureuse par ce moyen. Enfin, cette visée peut naturellement inclure celle de maintenir le lien, ce qui explique que la séquence de trois mots la plus utilisée dans le corpus soit je taime, avec 
3110 occurrences, sans compter les variantes comme jtm ni la binette $<3$, qui s'échangent aussi bien entre amants qu'entre amis et entre membres de la famille » (Vérine, 2013).

16. Exemples cités dans B. Vérine (2013).

17. S. Vernet (2010: ) montre, à partir de l'analyse de blogs sur Internet, que les remarques sur l'orthographe servent à disqualifier l'interlocuteur et ainsi à prendre un certain ascendant sur lui ( Commencez par corriger vos fautes d'orthographe, vous pourriez alors peut-être parler au nom des Français »).

18. Dans notre corpus de SMS, on ne trouve pas de récrimination sur l'orthographe ou l'écriture. On en reste au niveau des discours et des représentations à travers le questionnaire. En même temps, la thématisation sur l'orthographe dans les SMS peut-elle structurer les échanges quotidiens et familiers? Toujours est-il que toute anomalie orthographique est mise sur le compte de la technologie, comme s'il était toujours important de se dédouaner de toute erreur orthographique qui serait alors non intentionnelle ou de l'ordre de l'écriture SMS, délibérément choisie. On trouve alors les exemples suivants [Ha c'était marmotte Dsl c'est le correcteur d'orthographe], [draguer désolé c'est le correcteur d'orthographe :D], [Ok pas de souci (et désolé pour les fautes d'orthographe : saleté d'écriture intuitive!) Bisous bonne soirée à demain].

19. [Wesh gros! Et bien je sais pas si je pourrai parce que jai ptetre cours, enfin jte dirai ca ce soir ${ }^{\wedge \wedge}$ ].

20. ;-) ou ;) ou ^ pour souriant ou heureux ; : $p$ ou :P pour tirer la langue...

21. Par substitution (phonétisée [o] pour [eau], graphique [à +] pour [à plus]) : [J ai $\boldsymbol{u}<P R E_{-} 6>\mathbf{o}$ tel hier soir (pdt que monsieur recevai...)elle est ravie...tu as assure sur ce coup la,@+], par réduction (phonétisée avec acronymes [mdr, lol] ou troncations [ordi], [zou] pour [bisou], ou graphique [dc] pour [donc]), [Oui! J'etais trop deg!], [Bouh x.» bon allez espère que ta flemme s'est arrangée un peu.. Un zou], par suppression ou ajout graphique [Ahhh t'es trooooooooop fooorte !! :D :D j'y avais pas pensee mdrrrrr].

22. [Je n'arrive pas à dormir, mais je pense que toi si. Alors je vais en profiter pour essayer d'effectuer quelque chose que je nai plus pratiqué depuis quelques années... écrire une sorte de mini poème textoté, avec des rimes et des pensées ;) lol

Je ne sais jamais trop quoi t'écrire, mais j'aime le faire.

Je ne sais jamais trop quoi te dire, mais je ne peux me taire.

Je sais que j'aime te parler, mais j'ai juste peur de te lasser.

Ma batterie s'affaiblie et moi je ralenti, alors je vais te laisser te réveiller et moi aller me recoucher.

Bonne nuit $<$ PRE_5>:-)].

23. [Douki :3 alors gros gros bisous mon choupi chou!! :3 je t'aime fort fort fort $<3$ et tu me manques trop bcp bcp ... Fais de beau reves, et dors bien, je te fais un gros calinou :)]

\section{RÉSUMÉS}

Le lien avec la norme est au centre des perspectives sociolinguistiques puisqu'il rend compte des rapports de pouvoir et de légitimité entre les langues et les locuteurs de langue. D'un point de vue des locuteurs, les discours épilinguistiques sur ce qui est du «bien et beau parler » ou du «mal parler », qui disent combien «le français se perd», " on parle de plus en plus mal », « les élèves font de plus en plus de fautes » font partie de la doxa sur les pratiques langagières. L'usage quotidien des SMS, parce qu'il relève d'une pratique désormais répandue, utile voire ludique, aurait pu peut-être échapper aux carcans normatifs pour entrer dans une certaine modernité 
langagière qui dirait la diversité des usages, la liberté d'écrire et l'imaginaire scriptural. Or, les discours sur une orthographe, qui se verrait toujours plus détériorée par la pratique de l'écriture SMS, et a contrario mon propre étonnement face à la diversité des pratiques d'écriture SMS m'ont amenée à me pencher sur la représentation de la norme à travers les textos. Ainsi, à partir des réponses au questionnaire du projet Sud4Science et des commentaires métalinguistiques exprimés dans les textos, j'ai essayé de voir si les locuteurs reproduisent un discours normatif dominant ou si l'écriture SMS permet de développer un discours plus distancié et renouvelé par rapport à la norme.

A large part of sociolinguistic focuses on standard language and hence more unequal relations of power in society. The discourses of native speakers about "what is a good language" "what is a bad language" or about "The French language will be lost" and "Students make more and more spelling and grammatical mistakes" are very common characteristics of what is said about language practices. The widespread use of SMS messages could have escaped standard language control and, because the SMS writing is useful and fun, the result could even be a sort of language modernity finding freedom to write and the spelling imagination. But the discourses about the deteriorated spelling because of SMS use and contrary to that the diversity of SMS writing have led me to become interested in the representation of French standard about SMS use. In this way, based on the questionnaire of the Sud4Science project the analysis will focus on the gap between a dominant standard discourse and another more distanced.

INDEX

Mots-clés : sociolinguistique des discours, écriture SMS, orthographe, norme, rapports de pouvoir

Keywords : sociolinguitic and discourse, SMS writing, spelling, French standard, power relations

\section{AUTEUR}

\section{CLAUDINE MOÏSE}

Lidilem (609), université Stendhal, Grenoble 3 